Ann. Génét. Sél. anim., I972, 4 (3), 375-384.

\title{
CONSOMMATION ALIMENTAIRE DE PONDEUSES BLANCHES (I $i$ ) ET COLORÉES (ii)
}

\author{
P. MÉRAT et A. BORDAS \\ Laboratoire de Génétique factorielle \\ Centre national de Recherches zootechniques, I. N. R. A., \\ 78 - Jouy-en-Josas
}

\section{RÉSUMÉ}

Deux années successives (1970 et 1971), des poules en ponte, écloses à l'automne, de génotype $I i$ (absence de noir dans le plumage) ou ii (présence de noir), réparties également dans 6 familles de même père en 1970 et dans 7 en $197 \mathrm{I}$, ont été testées individuellement en cages, à partir de 8 mois d'âge, pour leur consommation alimentaire, leur ponte et leur variation de poids, pendant trois périodes successives de 28 jours. Les nombres de poules $I i$ et $i i$ utilisées étaient respectivement de 37 et 35 en 1970 , de 42 et 43 en 197 I.

Pour chaque poule, l'écart de la consommation observée par 28 jours à l'estimation obtenue à partir de la régression multiple sur le poids, la variation de poids et le poids d'œufs pondus (consommation " corrigée ") était calculé d'après la méthode de BYERLY (I94I). Un écart analogue, mais non corrigé pour la régression sur la variation de poids, était également considéré (consommation " partiellement corrigée ").

Les poules "blanches " $(I i)$ avaient une consommation "brute " inférieure à celle de leurs sœurs ou demi-sœurs " colorées " (ii) d'environ 6 p. Ioo en I970 $(P<0,05)$ et 2 p. roo en 197 , avec un poids corporel initial et une quantité d'œufs pondus très voisins en moyenne. La consommation " corrigée " ou " partiellement corrigée " était aussi inférieure pour les poules $I i$.

La différence de consommation moyenne entre les deux génotypes paraît se répéter régulièrement d'un jour à l'autre.

Sur un sous-échantillon abattu en fin d'expérience, en r97I, la quantité de graisse abdominale (en valeur absolue et en p. roo) est significativement plus élevée pour les poules colorées.

Le parallélisme de ces résultats avec nos données antérieures relatives au gène "blanc récessif " suggère un effet pléiotropique possible sur la consommation associée à l'inhibition ou à la réduction de la coloration.

\section{INTRODUCTION}

Dans deux expérimentations antérieures, en cages individuelles, sur des pondeuses âgées d'environ un an (ProD'HOMme et MÉRAT, I965; MÉRAT, I968), nous avions constaté que des poules blanches $c c$ avaient une consommation alimentaire 
" résiduelle " (corrigée pour le poids corporel, sa variation et le poids d'œufs pondus) inférieure de $4 \mathrm{p}$. Ioo environ à celle de leurs sœurs colorées $C c$. Ces dernières apparaissaient donc moins bonnes utilisatrices de l'aliment.

Ces résultats nous ont incités à faire une étude analogue pour un autre locus de coloration, en comparant la consommation et le rendement alimentaire de pondeuses $I i$ (blanches) et $i$ (colorées). Des données parallèles sur des coqs seront présentées séparément.

\section{MATÉRIEL ET MÉTHODES}

Le présent travail a été répété deux années, en I970 et en 197 I.

En 1970,72 pondeuses étaient utilisées au total, provenant d'un troupeau expérimental où divers gènes connus sont maintenus en ségrégation. Elles appartenaient à la descendance pedigree de 6 pères, chaque mère n'étant accouplée qu'à un coq. L'un des parents était hétérozygote pour le "blanc dominant " $I$, l'autre homozygote pour son allèle $i$, réalisant le croisement

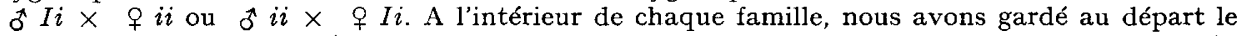
même nombre de poules $I i$ et $i i$, en nous efforçant en outre d'avoir un poids corporel initial voisin pour la moyenne des deux groupes.

Le génotype $I i$ correspondait à la présence d'un plumage entièrement blanc ou blanc avec une quantité variable de rouge; le plumage des animaux ii comportait des mélanines noires, allant du type "herminé " à plumage rouge au noir presque complet (présence du gène $\mathrm{E}$ ). Pour la commodité, nous désignons respectivement les phénotypes correspondants par "blanc " et " coloré ". La population était fixée, d'autre part, pour les allèles $s$ (doré lié au sexe) et $C$.

En I97I, un test similaire portait sur 85 pondeuses de la génération suivante du même troupeau, issues de 7 familles de même père, avec choix, ici encore, d'un nombre égal de poules $I i$ et $i i$ dans chacune.

Les deux années, les poules, écloses à l'automne, et élevées au sol en poussinière, étaient placées en batteries de ponte individuelles à l'âge de 20 semaines environ, puis transférées dans des cages adaptées au contrôle individuel de consommation à 8 mois. Une période d'adaptation d'un mois précédait la phase expérimentale. Cette dernière comprenait 3 périodes consécutives de 28 jours, s'étendant d'avril à juin. A la fin de chaque période, chaque pondeuse était pesée, ainsi que ses œufs et le poids total d'aliment ingéré ; une estimation individuelle était faite pour le gaspillage, d'ailleurs assez réduit dans nos conditions. L'aliment était donné sous forme de granulés de $2,5 \mathrm{~mm}$. Il correspondait à une formule "reproductrices ", à I6 p. Ioo de protéines et environ 2520 calories métabolisables $/ \mathrm{kg}$.

Les poules utilisées étaient celles dont toutes les performances étaient enregistrées dans les trois périodes expérimentales, à l'exclusion des mortes en cours d'expérience. Elles se répartissent en 37 o $I i$ et 35 o $i i$ en 1970, 42 I $i$ et 43 ii en 197 r.

En outre, au cours de chacune des deux années, pendant une brève durée à la fin de la dernière période (I I jours en 1970, I6 jours en I97I) on relevait la consommation journalière individuelle d'aliment et d'eau de boisson.

\section{RÉSULTATS}

\section{I. - Consommation "brute " (non corrigée)}

Les valeurs moyennes sur l'ensemble des trois périodes sont données, pour chaque année et chaque caractère mesuré, dans le tableau I. Pour fixer les idées, l'intensité de ponte était de l'ordre de 70 p. Ioo lors des deux expériences. Le détail de chaque période est omis, la différence entre génotypes $I i$ et $i i$ étant de même sens dans chacune des périodes successives, tant en I970 qu'en I97I. (En I970, 
l'écart dû au génotype diminuait de la première période à la troisième, mais nous avons vérifié qu'il n'y avait pas d'interaction significative entre génotype et période).

Une analyse de variance sur ces données (tabl. 2) indique en I970 un effet du génotype au locus $I$ significatif au seuil $5 \mathrm{p}$. Ioo pour la consommation d'aliment (d'environ $6 \mathrm{p}$. Ioo plus grande pour les poules ii) et le gain de poids.

En I97I, ces différences ne sont plus significatives, quoique la consommation d'aliment reste légèrement supérieure (d'environ 2 p. 100) pour les poules colorées. Quant aux différences entre familles, elles sont significatives pour tous les caractères en I970 (au seuil I p. Ioo) et pour trois d'entre eux (à l'exclusion du poids d'œufs pondus) en I 97 I. Nous avons omis, dans ce tableau, les variances " interaction ", un calcul approximatif ayant montré qu'aucune d'elles n'était significative.

TABLEAU I

Moyenne des performances "brutes " par génotype

\begin{tabular}{|c|c|c|c|c|c|c|}
\hline \multirow{2}{*}{\multicolumn{2}{|c|}{$\begin{array}{l}\text { Année } \\
\text { et génotype }\end{array}$}} & \multirow{2}{*}{$\begin{array}{c}\text { Aliment } \\
\text { consommé } / 28 \text { j. } \\
(\mathrm{g})\end{array}$} & \multicolumn{2}{|c|}{ Poids corporel } & \multirow{2}{*}{$\begin{array}{c}\text { Variation de } \\
\text { poids } / 28 \mathrm{j} \\
\text { (g) }\end{array}$} & \multirow{2}{*}{$\begin{array}{c}\text { Poids d'œufs } \\
\text { pondus } / 28 \mathrm{j} \\
\text { (g) }\end{array}$} \\
\hline & & & $\begin{array}{c}\text { en début } \\
\text { d'expérience } \\
\text { (g) }\end{array}$ & $\begin{array}{c}\text { moyen } \\
\text { (g) }\end{array}$ & & \\
\hline 1970 & $\begin{array}{l}I i \ldots \ldots \\
i i \ldots \ldots\end{array}$ & $\begin{array}{l}3335 \\
3540\end{array}$ & $\begin{array}{ll}2 & 069 \\
2 & 085\end{array}$ & $\begin{array}{l}2052 \\
2129\end{array}$ & $\begin{array}{r}-2,1 \\
+18,8\end{array}$ & $\begin{array}{l}966 \\
947\end{array}$ \\
\hline 1971 & $\begin{array}{l}\text { Ii...... } \\
\text { ii } \ldots . .\end{array}$ & $\begin{array}{l}3799 \\
3900\end{array}$ & $\begin{array}{l}2090 \\
2092\end{array}$ & $\begin{array}{l}2221 \\
2252\end{array}$ & $\begin{array}{r}+96,7 \\
+\quad 100,8\end{array}$ & $\begin{array}{l}977 \\
998\end{array}$ \\
\hline
\end{tabular}

TABLEAU 2

Analyse de variance sur les performances "brutes"

\begin{tabular}{|c|c|c|c|c|c|}
\hline \multirow[b]{2}{*}{ Source de variation } & \multirow{2}{*}{$\begin{array}{l}\text { Degrés } \\
\text { de liberté }\end{array}$} & \multicolumn{4}{|c|}{ Rapport F à la variance résiduelle et signification } \\
\hline & & $\begin{array}{l}\text { Consommation } \\
\text { d'aliment } / 28 \mathrm{j}\end{array}$ & $\begin{array}{c}\text { Poids } \\
\text { corporel moyen }\end{array}$ & $\begin{array}{c}\text { Variation } \\
\text { de poids } / 28 \mathrm{j}\end{array}$ & $\begin{array}{l}\text { Poids d'œufs } \\
\text { pondus } / 28 \mathrm{j}\end{array}$ \\
\hline 1970 & & & & & \\
\hline Génotype au locus I .... & 1 & $5,97 *$ & 1,74 & $4,87^{*}$ & 0,02 \\
\hline Familles ............ & 5 & $5,43^{* *}$ & $3,96 * *$ & $3,24 * *$ & $3,62^{* *}$ \\
\hline Résiduelle $\ldots \ldots \ldots \ldots$ & 60 & - & 一 & 一 & 一 \\
\hline 1971 & & & & & \\
\hline Génotypes $\ldots \ldots \ldots$ & 1 & 0,19 & 0,03 & 0,00 & 0,11 \\
\hline Familles $\ldots \ldots \ldots \ldots \ldots$ & 6 & $2,03^{*}$ & $2,20^{*}$ & $3,02^{* *}$ & 1,31 \\
\hline Résiduelle $\ldots \ldots \ldots \ldots$ & 71 & - & - & - & - \\
\hline
\end{tabular}

$*=\mathrm{P}<0,05$.

$* *=\mathrm{P}<0,01$. 
Accessoirement, nous avons vérifié qu'entre génotypes $I i$ et $i i$, dans cet échantillon, il n'existe pas de différence significative pour l'épaisseur et la coloration de la coquille, ainsi que pour la hauteur de l'albumen de l'œuf cassé sur une surface plane.

\section{2. - Consommation " corrigée 》}

D'après la méthode de BYERLY (I94I), nous avons établi les équations de régression multiple permettant d'estimer la consommation théorique d'une poule sur 28 jours $(\mathrm{A})$ exprimée en écart à la moyenne générale, en fonction de son poids corporel moyen $(\mathrm{P})$ de sa variation de poids $(\Delta \mathrm{P})$ et du poids d'œufs pondus $(\mathrm{O})$.

On obtient les équations suivantes, par année et par génotype, en omettant le terme constant $\left({ }^{1}\right)$ :

$$
\begin{aligned}
& \text { En I970 pour } I i: \mathrm{A}=62,30 \mathrm{P}^{0,5}+3,83 \Delta \mathrm{P}+0,66 \mathrm{O} \\
& \text { En I970 pour } i i: \mathrm{A}=66,63 \mathrm{P}^{0,5}+2,43 \Delta \mathrm{P}+0,72 \mathrm{O} \\
& \text { En I97I pour } I i: \mathrm{A}=76,63 \mathrm{P}^{0,5}+2,80 \Delta \mathrm{P}+0,75 \mathrm{O} \\
& \text { En I97I pour } i i: \mathrm{A}=49,29 \mathrm{P}^{0,5}+\mathrm{I}, 53 \Delta \mathrm{P}+0,9 \mathrm{I} \mathrm{O}
\end{aligned}
$$

Les deux années, il n'y a de différence significative entre génotypes pour aucun coefficient, de sorte que l'on peut établir une équation commune :

$$
\begin{aligned}
& \text { Pour r970: } \mathrm{A}=64,95 \mathrm{P}^{0,5}+3,04 \Delta \mathrm{P}+0,70 \mathrm{O} \\
& \text { Pour r97I : } \mathrm{A}=60,59 \mathrm{P}^{0,5}+2, \mathrm{II} \Delta \mathrm{P}+0,97 \mathrm{O}
\end{aligned}
$$

\begin{tabular}{|c|c|c|c|}
\hline $\begin{array}{c}\text { Source } \\
\text { de variation }\end{array}$ & $\begin{array}{c}\text { Degrés } \\
\text { de liberté }\end{array}$ & $\begin{array}{c}\mathrm{F} \\
\text { (rapport à la } \\
\text { variance résiduelle) }\end{array}$ & Signification \\
\hline 1970 & & & \\
\hline $\begin{array}{l}\text { Génotypes } \ldots \ldots \ldots \\
\text { Familles } \ldots . . . \\
\text { Interaction } \ldots \ldots \\
\text { Résiduelle } \ldots \ldots\end{array}$ & $\begin{array}{r}1 \\
5 \\
5 \\
60\end{array}$ & $\begin{array}{r}3,27 \\
11,38 \\
1,58 \\
-\end{array}$ & $\begin{array}{l}\mathrm{P} \simeq 0,05 \\
\mathrm{P}<0,01 \\
\quad \mathrm{NS} \\
\quad-\end{array}$ \\
\hline 1971 & & & \\
\hline $\begin{array}{l}\text { Génotypes } \ldots \ldots \ldots \\
\text { Familles } \ldots . . . \\
\text { Interaction } \ldots \ldots \\
\text { Résiduelle } \ldots \ldots\end{array}$ & $\begin{array}{r}1 \\
6 \\
6 \\
71\end{array}$ & $\begin{array}{l}0,10 \\
1,73 \\
1,29 \\
-\end{array}$ & $\begin{array}{l}\text { NS } \\
\text { NS } \\
\text { NS }\end{array}$ \\
\hline
\end{tabular}

La valeur du coefficient de corrélation multiple entre la consommation et les autres variables était de 0,80 en 1970 et de 0,76 en I97r.

\section{TABLEAU 3}

Analyse de variance pour la consommation "corrigée "

(Différence entre consommation observée et théorique, en grammes par 28 jours)

(1) L'exposant du poids a été pris à la valeur o,5, cette approximation mođifiant très peu, après vérification, les paramètres de l'équation par rapport à la valeur trouvée par le calcul. 
A partir de ces équations communes, nous avons calculé pour chaque poule la différence entre sa consommation réelle et sa consommation théorique. La moyenne de ces valeurs pour le génotype $i i$ dépasse celle des poules $I i$ de $86 \mathrm{~g}$ en I970, et de $52 \mathrm{~g}$ en I97I, ce qui représente respectivement 2,5 et I,3 p. Ioo de la consommation brute moyenne de l'année. Le tableau 3 contient l'analyse de variance correspondante.

Comme pour la consommation "brute ", la différence entre les génotypes $I i$ et ii, de même sens les deux années, n'est significative qu'en I970.

$\mathrm{Au}$ total, une meilleure utilisation " résiduelle " de l'aliment par les poules blanches est suggérée. Si, d'ailleurs, on détaille la comparaison entre génotypes $I i$ et ii à l'intérieur des familles, on s'aperçoit qu'en groupant les deux années, on obtient une moyenne inférieure pour les poules blanches dans Io familles sur I3: la probabilité correspondante est inférieure à 0,05 .

\section{3. - Consommation "partiellement corrigée " (pour le poids corporel et la ponte)}

Il n'existe pas de différence entre les deux phénotypes, blanc et coloré, dans le poids corporel initial, choisi de toutes façons aussi voisin que possible, ni dans la quantité d'œufs produite, ce dernier point étant confirmé par d'autres données (MÉRAT, I962 et résultats non publiés). L'ingestion alimentaire paraît être le phénomène premier différenciant les deux groupes; la seule autre différence concerne le gain de poids et peut en être considérée comme une conséquence naturelle.

\section{TABLEAU 4}

Analyse de variance, consommation "partiellement corrigée "

(Pour le poids corporel et la ponte)

\begin{tabular}{|c|c|c|c|}
\hline $\begin{array}{c}\text { Source } \\
\text { de variation }\end{array}$ & $\begin{array}{l}\text { Degrés } \\
\text { de liberté }\end{array}$ & $\begin{array}{c}\mathrm{F} \\
\text { (rapport à la } \\
\text { variance résiduelle) }\end{array}$ & Signification \\
\hline 1970 & & & \\
\hline $\begin{array}{l}\text { Génotypes }-\ldots \ldots \\
\text { Familles } \ldots \ldots \ldots \\
\text { Interaction } \ldots \ldots \\
\text { Résiduelle } \ldots \ldots\end{array}$ & $\begin{array}{r}1 \\
5 \\
5 \\
60\end{array}$ & $\begin{array}{c}7,25 \\
6,55 \\
1,93 \\
-\end{array}$ & $\begin{array}{c}\mathrm{P}<0,01 \\
\mathrm{P}<0,001 \\
\mathrm{NS} \\
-\end{array}$ \\
\hline 1971 & & & \\
\hline $\begin{array}{l}\text { Génotypes } \ldots \ldots \ldots \\
\text { Familles } \ldots \ldots \ldots \\
\text { Interaction } \ldots \ldots \\
\text { Résiduelle } \ldots \ldots\end{array}$ & $\begin{array}{r}1 \\
6 \\
6 \\
71\end{array}$ & $\begin{array}{l}0,06 \\
1,36 \\
1,23 \\
-\end{array}$ & $\begin{array}{l}\text { NS } \\
\text { NS } \\
\text { NS } \\
-\end{array}$ \\
\hline
\end{tabular}

Nous avons donc, complémentairement, comparé les génotypes $I i$ et $i i$ en prenant pour variable la consommation alimentaire corrigée seulement pour le poids corporel moyen et le poids d'œufs pondus. Cela revenait à utiliser des équations de 
régression semblables aux précédentes, mais en omettant le terme contenant la variation de poids $\Delta \mathrm{P}$.

L'excès de consommation par 28 jours des poules $i i$ sur les $I i$, estimé de cette façon, est égal à $\mathrm{I} 64 \mathrm{~g}$ pour I970 et à $60 \mathrm{~g}$ pour I97I (soit respectivement 4,8 et I, 6 p. roo de la consommation moyenne). Une analyse de variance révèle que l'effet " génotype " sur la consommation ainsi corrigée partiellement est significatif au seuil I p. Ioo en I970, et non significatif en I97I (tabl. 4).

Les présents résultats présentent une analogie avec nos données antérieures relatives au locus $C$ (PROD'Homme et MÉRAT, I965; MÉRA'T, I968). Là également, les poules blanches $(c c)$ avaient une consommation "corrigée " moindre que les colorées, et il en était de même de leur gain de poids pendant les périodes expérimentales, alors que, dans l'ensemble, il n'y avait pas de différence significative concernant la ponte et le poids corporel initial. Nous avons, par suite, réanalysé nos résultats publiés en Ig68, pour le "blanc récessif ", en considérant ici aussi la variable "consommation corrigée sauf pour la variation de poids" : elle est, en moyenne, inférieure de $\mathrm{I} 86 \mathrm{~g}$ pour les poules $c c$, la différence représentant plus de 5 p. Ioo de la consommation brute moyenne de l'ensemble de l'échantillon. Une analyse de variance avec les facteurs "génotype " et "famille " indique que l'écart moyen entre génotypes pour cette variable est significatif au seuil $5 \mathrm{p}$. Ioo (rapport $\mathrm{F}$ à la variance résiduelle égal à 5,98 pour I et 45 d.1.).

De même, dans nos résultats publiés en I965, la consommation partiellement corrigée est de $239 \mathrm{~g}$ plus faible pour les poules blanches $(c c)$ que pour les colorées (environ 6 p. Ioo de la consommation brute moyenne).

\section{4. - Etat d'engraissement en fin d'expérience}

En I97I, après la fin de la dernière période expérimentale, I5 poules de génotype $I i$ et $\mathrm{I}_{5}$ ii ont été choisies parmi les survivantes, par couples de sœurs ou demisours, l'une $I i$, l'autre $i$, de poids voisin en début de test (en moyenne $2036 \mathrm{~g}$ pour les $I i$ et $2033 \mathrm{~g}$ pour les $i i$ dans cet échantillon). Pour ces poules, le foie et la graisse abdominale (principalement autour du gésier et de la bourse de Fabricius) ont été pesés (tabl. 5).

\section{TABLEAU 5}

Poids de la graisse abdominale et du foie pour un sous-échantillon de poules de génotypes $\mathrm{Ii}$ et ii

\begin{tabular}{|c|c|c|c|c|c|c|}
\hline \multirow{2}{*}{ Génotypes } & \multicolumn{3}{|c|}{ Graisse abdominale $(\mathrm{g})$} & \multicolumn{3}{|c|}{ Foie $(g)$} \\
\hline & $\bar{x}$ & $s^{2}$ & $t\left({ }^{1}\right)$ & $\bar{x}$ & $s^{2}$ & $t$ \\
\hline $\begin{array}{l}I i \\
i i\end{array}$ & $\begin{array}{l}163,3 \\
197,5\end{array}$ & $\begin{array}{l}2928 \\
5698\end{array}$ & $2,14^{*}$ & $\begin{array}{l}41,3 \\
43,8\end{array}$ & $\begin{array}{r}42,9 \\
114,0\end{array}$ & 0,80 \\
\hline
\end{tabular}

(1) Le test est applicable, le rapport des variances n'étant pas significativement différent de l'unité. 
En valeur absolue, le poids de graisse abdominale est significativement plus élevé pour les poules colorées. Il en est de même en pourcentage $(7,3 \mathrm{p}$. Ioo pour les 우으 $i$ contre $8,0 \mathrm{p}$. Ioo pour les $i i)$, malgré une différence dans le poids corporel atteint à 1'abattage ( $2237 \mathrm{~g}$ contre $246 \mathrm{I} \mathrm{g}$ ). Le sous-échantillon choisi se trouve d'ailleurs par hasard présenter un gain de poids moyen supérieur à celui de l'ensemble.

Il est à rappeler que, lors de notre premier test de consommation individuelle sur des poules $C c$ et $c c$ (ProD'homme et MÉRAT, I965) nous avions trouvé sur un échantillon d'effectif réduit un poids supérieur de graisse abdominale pour les poules colorées.

Il est vraisemblable que la différence de gain de poids obtenue dans l'ensemble entre poules $I i$ et $i i$ (de même qu'entre poules $C c$ et $c c$ ) est en majeure partie une différence dans l'importance des tissus gras élaborés. Dans 1'échantillon où la graisse abdominale a été pesée, la corrélation entre le poids de celle-ci et le gain de poids enregistré pendant l'ensemble du contrôle de consommation est égale à $+0,6 \mathrm{I}$ $(\mathrm{P}<\mathrm{O}, \mathrm{OI})$.

\section{5. - Consommation d'eau}

La consommation journalière moyenne d'eau pendant une courte période en fin d'expérience a été, en I970, de $278 \mathrm{~g}$ pour les poules $I i, 338 \mathrm{~g}$ pour les $i i$; les valeurs correspondantes en I97I étaient $338 \mathrm{~g}$ et 3 I $6 \mathrm{~g}$. Quoiqu'elle représente un pourcentage relativement élevé en I970, la différence n'était significative dans aucune des deux années, du fait de la grande variabilité individuelle existant pour ce caractère.

Nous avons observé, au niveau individuel, 1'existence d'une corrélation positive entre consommation d'eau et consommation alimentaire " résiduelle ". On vérifie en tout cas que les différences intra-année de consommation d'eau entre génotypes $I i$ et $i i$ (d'ailleurs non significatives et de sens contraire suivant 1'année) ne pourraient suffire à rendre compte des différences entre ces génotypes pour la consommation " résiduelle " d'aliment.

\section{6. - Consommation journalière d'aliment}

Il est intéressant, accessoirement, d'observer la consommation journalière moyenne des poules colorées et blanches en I970 (fig. I) et I97I (fig. 2).

En I970, II jours sur II, la consommation moyenne des poules ii est supérieure à celle des $I i$; en I97I, elle la dépasse 12 fois sur I6, la différence étant très faible ( $\mathrm{r}$ à $3 \mathrm{~g}$ ) pour trois des quatre autres jours.

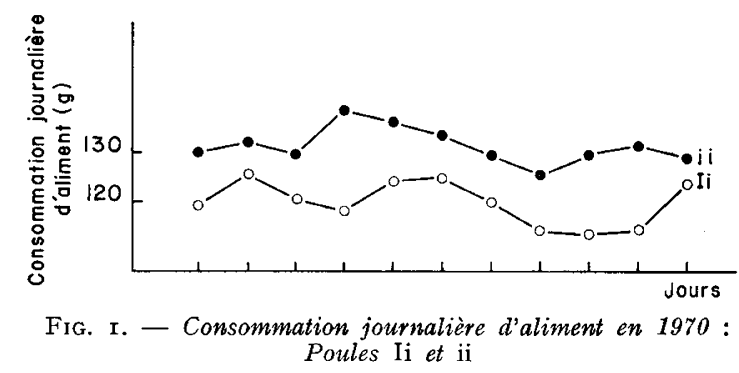


En considérant les différences d $j$ entre consommation moyenne au jour $j$ des poules $i$ et des poules $I i$ comme des variables normales indépendantes, on constate, pour les deux années, une valeur moyenne de ces différences très significativement positive : En I970, cette valeur est voisine de + II,I g d'où $t=7,60$ avec II d.1. $(\mathrm{P}<0,00 \mathrm{I})$; en I97I, la valeur trouvée, égale à $+4,4 \mathrm{~g}$, est significative au seuil I p. Ioo $(t=3,47$ avec I6 d.1.).

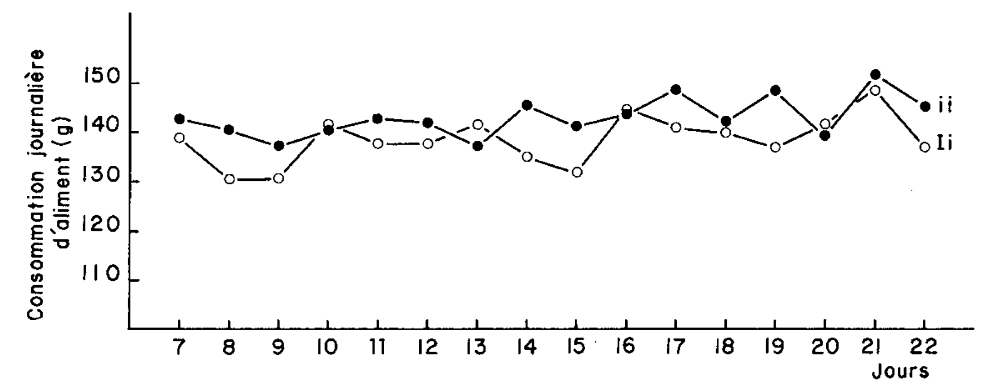

FIG. 2. - Consommation journalière d'aliment en $19 \pi 1$ :

Poules Ii et ii

La production moyenne d'œufs des deux génotypes était extrêmement voisine, les deux années, pendant la période correspondante, et ne pouvait (de même que sur l'ensemble des périodes de 28 jours) rendre compte de la différence de consommation globale. Il en est de même pour les poids corporels moyens, voisins dans la période en cause en I97I, le poids supérieur des poules ii pendant le contrôle journalier en I970 ne pouvant expliquer qu'une faible partie de la différence de consommation observée.

\section{DISCUSSION ET CONCLUSTONS}

Des différences entre familles se retrouvent en I970 et I97I pour la consommation brute, en I970 pour la consommation " corrigée ", de même que dans nos essais antérieurs (PROD' HOMME et MÉRAT, I965; MÉRAT, I968). Il existe donc une variabilité génétique appréciable pour la consommation indépendamment du poids, du gain de poids et de la ponte. Ceci, à notre connaissance, a rarement été mis en évidence.

De plus, l'un des facteurs génétiques responsables de la variation de la consommation alimentaire indépendamment du poids corporel et de la ponte parait, à la lumière de l'ensemble de nos résultats, associé au locus $I$ de coloration du plumage. Si la différence globale entre les génotypes $I i$ et $i$, pour la consommation brute ou corrigée, n'est pas significative en I97I, elle est de même sens les deux années, dans chaque période de 28 jours intra-année, et à l'intérieur de la grande majorité des familles utilisées. En outre, en I970 et même en 1971, la régularité avec laquelle, jour par jour, se répète la différence entre génotypes est une autre indication de même que l'inégalité observée dans l'état d'engraissement sur un échantillon restreint. 
A partir du parallèle avec les résultats déjà cités relatifs au locus $C$ (poules "blanches " consommant moins que les " colorées " dans les deux cas), il est tentant de supposer que les effets associés aux deux loci représentent une action pléiotropique, car sinon l'on devrait supposer dans chaque cas l'existence d'un linkage relativement étroit (les gènes étudiés étant maintenus en ségrégation depuis plus de Io générations) entre l'allèle responsable de l'absence de pigmentation et un facteur de moindre consommation.

Des résultats non publiés nous suggèrent d'ailleurs que, de même, la consommation des poules à noir restreint dans le plumage est un peu inférieure à celle de leurs sœurs à noir étendu (présence du gène $E$ ).

Aux gènes en question est associée une légère différence de rendement alimentaire, à en juger par la consommation corrigée pour les trois facteurs poids, variation de poids et poids d'œufs pondus. Cependant, ceci peut n'être qu'une conséquence de l'ingestion plus grande d'aliment, et, simultanément, du gain de poids supérieur et de la conversion en graisse d'une partie plus importante de cet aliment, à poids initial et ponte égaux, chez les poules colorées. Ainsi, avec les gènes $I$ et $C$, nous aurions localisé des facteurs génétiques de comportement alimentaire, ayant un effet sur l'appétit. Bien entendu, cet effet peut être direct ou indirect. Il pourrait s'agir d'un effet " social ", par exemple d'une différence de hiérarchie entre poulettes blanches et colorées en fin de période d'élevage au sol en poussinière, ayant influé sur leurs habitudes alimentaires (quoique cette période soit à vrai dire assez éloignée de la période expérimentale). On pourrait aussi penser à un effet physique (thermorégulation différente ?) ou encore à une modification légère de la régulation de l'appétit d'ordre biochimique ; dans ce cas, l'on ne peut affirmer a priori laquelle, de la différence d'appétit ou d'engraissement, est la cause de l'autre.

D'autres différences, peu considérables mais diverses, ont été rencontrées entre génotypes opposés pour la pigmentation du plumage : croissance (cf. revues par SMYTH, I969; MÉRAT, I970 a), épaisseur et coloration des coquilles d'œufs (MÉRAT, I970 b), poids des œufs (MÉRAT données non publiées). On pourrait en chercher une interprétation à la lumière d'une consommation alimentaire inégale.

Sous l'angle pratique, si le caractère pléiotropique d'associations entre coloration du plumage et capacité d'ingestion d'aliment est confirmé, quelques conséquences en seraient à suggérer pour l'utilisation des pondeuses suivant qu'il s'agit de souches blanches ou colorées. Si les premières tendent à sous-consommer légèrement par rapport aux autres, elles peuvent être des productrices d'œufs ou de poussins plus rentables : Il est permis de se demander si cela a contribué au maintien, dans des races de ponte comme la Leghorn et la Wyandotte, de la seule variété blanche à l'échelon commercial ? D'autre part, il pourrait alors se révéler avantageux de rationner des poulettes blanches un peu moins strictement que les colorées, même à format égal, et peut-être de leur donner, au stade pondeuse, un aliment légèrement plus riche en certains composants.

Enfin, un autre aspect de nos résultats est la possibilité qu'ils suggèrent d'une appréciation de l'indice de consommation individuel des pondeuses sur une période assez courte, en particulier si la consommation est enregistrée jour par jour : nous détaillerons ceci dans un autre article. 


\section{REMERCIEMENTS}

Nous remercions M. Guillaume (Station de Recherches avicoles, 37 - Nouzilly) de ses remarques et critiques très constructives concernant ce manuscrit.

\section{SUMMARY}

FEED CONSUMPTION OF WHITE ( $I$ i ) AND COLORED (ii) LAYING HENS.

Two successive years (I970 and 197I), laying hens, hatched in autumn, of the genotypes $I i$ (absence of black in the plumage) or $i i$ (presence of black), sampled in equal numbers among 6 sire families in 1970 and 7 in I97I, were individually tested in cages from 8 months of age for feed consumption, egg production and weight gain or loss, during three consecutive 28-day periods. The numbers of $I i$ and $i i$ hens utilized were respectively 37 and 35 in 1970,42 and 43 in $197 \mathrm{I}$.

For each hen, the deviation of observed feed consumption per 28 days from the expectation given by the multiple regression equation on weight, weight variation and weight of eggs laid (" corrected " consumption) was calculated according to ByERLy's method (I94I). A similar deviation, uncorrected for regression on weight gain, was also considered (" partly corrected " consumption).

"White " (Ii) hens had a lower " uncorrected " feed consumption than their "colored "(ii) full- or half-sisters, by about 6 per cent in $1970(\mathrm{P}<0,05)$ and 2 per cent in $197 \mathrm{I}$, with a very close initial body weight and eggs production on the average during the test. The "corrected" or " partly corrected " consumption was also somewhat lower for $I i$ hens.

The average difference of feed ingestion between the two genotypes seems to be regularly repeated day after day.

In a sub-sample slaughtered at the end of the experimental period in I97 $\mathrm{r}$, the amount (absolute or relative) of abdominal fat is significantly higher for colored hens.

The parallelism of theses results with our previous data concerning the "recessive white " gene suggests a possible pleiotropic reduction of feed consumption associated with an inhibition or reduction of plumage pigmentation.

\section{RÉFÉRENCES BIBLIOGRAPHIQUES}

Byerly T. C., r94I. Feeds and other costs of producing market eggs. Univ. Maryland Agric. Exp. Sta: Bull. $\mathrm{n}^{\circ} \mathrm{A} \mathrm{I}$.

MÉrat P., 1962. Quelques relations entre caractères extérieurs à hérédité simple et productivité. C. $R$. $X I I^{\mathrm{e}}$ Congrès mondial d'Aviculture, 7, I-76.

MÉrat P., rg68. Consommation alimentaire de pondeuses $C c$ et $c c$. Ann. Zootech. 17, 337-339.

MÉrat P., I970a. Mendelian genetics and selection for quantitative traits in poultry : Results and perspectives. World Poult. Sci. J. 26, 57I-586.

MÉrat P., I97o $b$. Gènes à effet visible et coloration ou épaisseur des coquilles d'œufs. Ann. Génét. Sél. anim., 2, 263-267.

Prod'homme J., Mérat P., x965. Consommation alimentaire chez des pondeuses de génotype $C c$ et $c c$ issues de plusieurs familles. Ann. Zootech. 14, 34I-350.

Sмутн J. R., Jr, 1969. Relationship between genes affecting melanin pigmentation and other traits in the fowl. World's Poult. Sci. J., 25, 6-I4. 